\title{
EFECTIVIDAD DE UN TALLER DE CAPACITACIÓN A RESIDENTES PARA EL DIAGNÓSTICO DE ENFERMEDAD ARTERIAL PERIFÉRICA
}

\author{
Martín Alejandro Flores-Méndez ${ }^{*}$, Miguel Ángel Rodríguez-Castellanos², Adán Irán Vázquez-Burgo ${ }^{3}$, \\ Issa Gil-Alfaro ${ }^{4}$ y Balkis Vera Cruz-Mendoza ${ }^{3}$ \\ Especialista en medicina familiar, Puebla, México; ${ }^{2}$ Especialista en Angiología y Cirugía Vascular; ${ }^{3}$ Especialista en medicina familiar; \\ ${ }^{4}$ Maestra en investigación clínica. IMSS, Veracruz, México
}

RESUMEN: Objetivo general: Evaluar la efectividad de un taller de capacitación dirigido a médicos residentes de medicina familiar para el diagnóstico oportuno de enfermedad arterial periférica (EAP) mediante el método índice tobillo-brazo (ITB). Material y métodos: Se realizó una intervención educativa en la Unidad de Medicina Familiar (UMF) 66 en Xalapa (Veracruz) de septiembre a noviembre de 2017. Se incluyeron en el estudio todos los residentes de medicina familiar adscritos a esa unidad que, previo consentimiento informado, aceptaron acudir al $100 \%$ de las sesiones programadas. No fueron incluidos aquéllos con evaluaciones incompletas. Se realizó una medición previa y otra posterior al curso de capacitación. Se evaluó el grado de conocimiento sobre la EAP y el método ITB mediante un instrumento elaborado por un asesor experto (angiólogo) y un médico residente de medicina familiar. El estudio fue aprobado por el Comité de Investigación y Ética. Se utilizaron frecuencias absolutas y relativas, y las pruebas de Rangos de Wilcoxon, U de Mann Whitney y de Kruskal-Wallis para la evaluación de las diferencias entre las evaluaciones iniciales y finales al 95\% de confianza. Resultados: Los conocimientos se incrementaron significativamente $(\mathrm{p}<0.0001)$ entre la mediana del resultado global en la evaluación inicial (60, rango intercuartilar [RIC]: 51-68) y la evaluación final (97,
EFFECTIVENESS OF A TRAINING WORKSHOP FOR RESIDENTS FOR THE DIAGNOSIS OF PERIPHERAL ARTERIAL DISEASE

ABSTRACT: To evaluate the effectiveness of a training workshop aimed at family medicine residents for the opportune diagnosis of peripheral arterial disease (PAD) using the ankle-brachial index (ABI) Materials and Methods: An educational intervention was carried out at Family Medicine Unit No. 66 in Xalapa, Veracruz, Mexico from September to November 2017. All family medicine residents assigned to the unit accepted, after obtaining informed consent, to attend $100 \%$ of the programmed sessions. Those with incomplete evaluations were eliminated. A pre- and post-measure of the training course was performed. The degree of knowledge of PAD and the ABI were assessed using an instrument developed by an expert advisor (an angiologist) and a family medicine resident. The protocol was approved by the Ethics and Research Committee. Absolute and relative frequencies, and the Wilcoxon, MannWhitney- $U$ and the Kruskal Wallis tests were used to determine differences between the initial and final evaluations with a 95\% confidence interval. Results: Knowledge increased significantly $(\mathrm{p}<0.0001)$ between the median of the overall result in the initial evaluation (60, RIC 51-68) and the final evaluation (97, RIC 94-100). Participants improved in the areas of general knowledge, adequate procedure for $\mathrm{ABI}$ measurement
Correspondencia:

*Martín Alejandro Flores-Méndez

E-mail:marlex7@hotmail.com
Fecha de recepción: 10-07-2018

Fecha de aceptación: 23-10-2018

DOI: 10.24875/RMF.18000099 
RIC: 94-100). Se mejoró en los rubros de conocimiento general, en el procedimiento adecuado para la medición del ITB y en el cálculo del ITB ( $\mathrm{p}<$ 0.0001). Conclusiones: El taller fue efectivo para el diagnóstico de la EAP mediante el método ITB en los residentes de medicina familiar.

Palabras clave: Programa de entrenamiento. Estudiantes de medicina. Enfermedad arterial periférica. Índice tobillo-brazo. and ABI calculation $(\mathrm{p}<0.0001)$. Conclusions: The workshop was effective for the diagnosis of PAD using the ankle-brachial index. This model could be applied in medical units as an intervention to reduce the prevalence of PAD morbidity and mortality. (Rev Mex Med Fam. 2019;6:14-20)

Corresponding author: Martín Alejandro FloresMéndez, marlex7@hotmail.com

Key words: Training program. Medical students. Peripheral artery disease. Ankle-brachial index.

\section{INTRODUCCIÓN}

La EAP es una enfermedad altamente prevalente que afecta a millones de personas en todo el mundo ${ }^{1}$, y se encuentra íntimamente asociada a la enfermedad cardiovascular y cerebral -debido a su sustrato anatomopatológico- y a la aterosclerosis, con la consecuente oclusión o estenosis de la luz arterial ${ }^{2}$. Por ello es frecuente que pacientes con EAP presenten alteraciones coronarias (infarto de miocardio) o cerebrales (evento vascular cerebral), enfermedades que se encuentran dentro de las primeras causas de morbimortalidad en México. Asimismo, es una enfermedad «silenciosa» que amerita un diagnóstico efectivo y oportuno ${ }^{3,4}$.

El método diagnóstico recomendado para la EAP es el ITB, ya que es una herramienta estandarizada, económica, no invasiva, objetiva y de fácil aplicación en la atención de primer nivel. Un ITB patológico es un predictor independiente de riesgo y mortalidad cardiovascular, además de un factor predictivo de lesión de órganos blanco (renal, cerebral, corazón) ${ }^{5}$. Esta prueba posibilita un diagnóstico oportuno y permite acceder a un tratamiento oportuno y a una derivación temprana a otras especialidades médicas (angiología y cardiología) ${ }^{6}$.

El método ITB es una prueba altamente específica y sensible siempre que sea realizada por profesionales capacitados. Desafortunadamente, existen muy pocos estudios enfocados a realizar programas de entrenamiento para la enseñanza adecuada del método ITB, por lo que se carece de una capacitación formal, motivo por el cual los profesionales de la salud lo utilizan poco y $\mathrm{mal}^{7,8}$.

En la literatura médica hay pocos estudios que hayan abordado el impacto de un programa de capacitación. En un estudio con médicos residentes, la capacitación se realizó por medio de ayuda audiovisual (video en internet) con evaluación a través de casos clínicos, pero sin asesor experto. La evaluación se realizó a través del porcentaje mediante el número de aciertos adquiridos antes y después del video (no se especifica el tiempo de la capacitación $)^{9}$.

En otro estudio en estudiantes de medicina se evaluó cómo realizar el ITB. La capacitación fue teórico-práctica, sin cuestionario de evaluación ni lista de cotejo. En este caso, la evaluación se llevó a cabo comparando a los estudiantes con el cirujano vascular ${ }^{10}$. En otro estudio realizado a médicos generales la capacitación fue teórica sobre la EAP y la medición del ITB, pero también sin cuestionarios de capacitación ni lista de cotejo. En este estudio se evaluó con médicos generales que no tomaron el curso de capacitación ${ }^{11}$. En estos últimos dos estudios sus capacitaciones fueron de aproximadamente seis meses.

En nuestro estudio, en cambio, la capacitación fue de una sola sesión $(2 \mathrm{~h})$ y 
teórico-práctica, con instrumento de evaluación y lista de cotejo para realizar el ITB, y soporte con un asesor experto (cirujano vascular).

Considerando que la EAP es una enfermedad con graves repercusiones médicas y económicas, y que en una investigación realizada en el año 2014 en la UMF 66 se reportó un nulo diagnóstico en los pacientes ${ }^{12}$, se consideró prioritario elaborar y evaluar la efectividad de un taller de capacitación para el diagnóstico oportuno de insuficiencia arterial periférica con el uso del método ITB, teniendo presente los conocimientos, el procedimiento adecuado para su realización y la evaluación e interpretación del cálculo del ITB.

\section{MATERIAL Y MÉTODOS}

Se llevó a cabo una intervención educativa en la UMF 66 de Xalapa (Veracruz) en el año 2017 dirigida a la totalidad de los residentes de la especialidad de medicina familiar adscritos a esa unidad que, previo consentimiento informado, aceptaron acudir al $100 \%$ de las sesiones programadas. No se incluyeron aquéllos con evaluaciones o cuestionarios incompletos ni a los que se encontraban en rotaciones externas a la unidad (a la mitad de los residentes de tercer año se les realizó el taller antes de que se fueran a su servicio social). El programa del taller se elaboró con la participación conjunta de un asesor experto (cirujano vascular angiólogo) y un instructor (un médico residente), capacitado por el propio asesor, quien supervisó las sesiones, las cuales se enfocaron en grupos reducidos de 8-10 residentes, con una duración total de 130 min que comprendieron: a) evaluación inicial con aplicación del instrumento, explicación del taller y contenido temático, capacitación teórica por medio de diapositivas, video y material didáctico relativo a la EAP y el ITB (50 min); b) enseñanza práctica del método ITB con paciente, que se evaluó con lista de cotejo utilizando los siguientes materiales: un esfigmomanómetro (manual o digital), un equipo Doppler pulsado con transductor de $10 \mathrm{MHz}$ y gel para ultrasonidos (60 min), y c) aplicación del cuestionario para la evaluación final y clausura del taller (20 min).

Los residentes efectuaron la determinación del ITB en 54 pacientes de la siguiente manera: se indicó al paciente descansar por 5 min en una cama en posición decúbito supino; se buscó la arteria braquial del lado derecho para colocar gel sobre ésta y después, con el transductor Doppler y en un ángulo aproximado de $45^{\circ}$, se colocó en la zona que produjo el sonido más audible, para posteriormente insuflar la presión del manguito al menos $20 \mathrm{mmHg}$ por encima de la presión arterial sistólica (PAS) hasta la desaparición del flujo y después desinflar hasta la reaparición de la señal del flujo, registrar la presión sistólica más alta y anotarla. Este mismo procedimiento se realizó en las cuatro extremidades en el siguiente orden: arterias braquial, pedia y tibial posterior derechas, arterias tibial posterior, pedia y braquial izquierdas. Para el cálculo del ITB se utilizó la PAS braquial más elevada de ambos brazos y la más alta de una de las arterias del tobillo. El ITB derecho fue el cociente entre el valor de la PAS más elevada del tobillo derecho y el valor más alto de la PAS de ambas arterias braquiales; y para el izquierdo se siguió el mismo procedimiento. De los dos valores, el de menor cuantía es el que delimitó la existencia de EAP. Se diagnosticó EAP en aquellos pacientes con un ITB $<0.9$.

Se evaluó el porcentaje de respuestas correctas que obtuvo cada participante del total de reactivos. Se consideró como aprobado al participante si obtenía más del $60 \%$ de respuestas correctas. El instrumento utilizado constó de 35 reactivos o ítems (calificación del 100\%) que evaluaban tres áreas: conocimiento general de la EAP e ITB, procedimiento adecuado para su realización y evaluación del cálculo e interpretación del ITB, además de una lista de cotejo para evaluar la realización del ITB en los pacientes. Se analizaron y compararon los resultados obtenidos por los participantes por grado de residencia. El instrumento y la lista de 
cotejo fueron realizados por el asesor experto y el médico residente de medicina familiar según los lineamientos de la American College of Cardiology Foundation/American Heart Association ${ }^{4}$. No se consideró necesario medir la concordancia entre residente y experto, dado que ambos conjuntamente realizaron las evaluaciones mediante el instrumento y la lista de cotejo. Se consignaron también variables sociodemográficas y familiares.

\section{Consideraciones éticas}

Este estudio fue valorado y aprobado por el Comité Local de Investigación y Ética, otorgando Registro SIRELCIS F-2017-3005-85. Se solicitó consentimiento informado, se notificó a todos los participantes que se realizaría una evaluación inicial y otra final, y se les especificó que los resultados obtenidos no tendrían repercusión alguna en sus calificaciones.

\section{Análisis estadístico}

Para la descripción de las variables del estudio se utilizaron frecuencias absolutas y relativas. Para las evaluaciones inicial y final se utilizaron mediana y RIC, dado que no se obtuvo una distribución normal (prueba de Shapiro-Wilk y/o prueba de Kolmogorov-Smirnov $\mathrm{p}<0.05)$. Para evaluar la mediana de las diferencias entre la evaluación inicial y la final para muestras dependientes se utilizó la prueba de rangos de Wilcoxon, para obtener diferencias en promedios entre géneros la prueba de la $U$ de Mann-Whitney y para los grados académicos de residencia se utilizó la prueba de Kruskal-Wallis. Se consideró estadísticamente significativo un valor de $\mathrm{p}<0.05$. Se utilizó el software estadístico IBM SPSS, versión 22.

\section{RESULTADOS}

En el curso de la capacitación para el método ITB participaron 54 residentes de medicina familiar (100\%) con las siguientes características: predominio de mujeres, solteros, edad de 25 a 30 años, pertenecientes
Tabla 1. Características sociodemográficas de los residentes de medicina familiar

\begin{tabular}{|c|c|c|}
\hline & Frecuencia & Porcentaje \\
\hline Total de residentes & 54 & $100 \%$ \\
\hline \multicolumn{3}{|l|}{ Sexo } \\
\hline Masculino & 21 & $39 \%$ \\
\hline Femenino & 33 & $61 \%$ \\
\hline \multicolumn{3}{|l|}{ Estado civil } \\
\hline Soltero & 32 & $59 \%$ \\
\hline Casado & 14 & $26 \%$ \\
\hline Unión libre & 8 & $15 \%$ \\
\hline \multicolumn{3}{|l|}{ Edad } \\
\hline 25-30 años & 31 & $57 \%$ \\
\hline 31-35 años & 16 & $30 \%$ \\
\hline 36-40 años & 7 & $13 \%$ \\
\hline \multicolumn{3}{|l|}{ Composición familiar } \\
\hline Nuclear simple & 41 & $76 \%$ \\
\hline Nuclear numerosa & 4 & $7 \%$ \\
\hline Reconstruida & 1 & $2 \%$ \\
\hline Monoparental & 4 & $7 \%$ \\
\hline Monoparental extendida & 1 & $2 \%$ \\
\hline Extensa & 1 & $2 \%$ \\
\hline No parental & 2 & $4 \%$ \\
\hline \multicolumn{3}{|l|}{ Demografía } \\
\hline Urbana & 49 & $91 \%$ \\
\hline Suburbana & 5 & $9 \%$ \\
\hline \multicolumn{3}{|l|}{ Desarrollo familiar } \\
\hline Moderna & 43 & $80 \%$ \\
\hline Tradicional & 11 & $20 \%$ \\
\hline \multicolumn{3}{|l|}{ Ocupación del jefe de familia } \\
\hline Campesino & 1 & $2 \%$ \\
\hline Obrero & 1 & $2 \%$ \\
\hline Empleado & 5 & $9 \%$ \\
\hline Comerciante & 3 & $6 \%$ \\
\hline Profesionista & 44 & $81 \%$ \\
\hline \multicolumn{3}{|l|}{ Integración familiar } \\
\hline Integrada & 47 & $87 \%$ \\
\hline Desintegrada & 7 & $13 \%$ \\
\hline \multicolumn{3}{|l|}{ Pobreza familiar } \\
\hline Sin evidencia & 53 & $98 \%$ \\
\hline Baja & 1 & $2 \%$ \\
\hline
\end{tabular}

a familia nuclear simple, urbana, integrada, moderna y sin pobreza familiar (Tabla 1 ). 
Tabla 2. Evaluaciones inicial y final del taller de capacitación para el diagnóstico de EAP

\begin{tabular}{|c|c|c|c|}
\hline \multirow{2}{*}{$\begin{array}{l}\text { Áreas a evaluar. } \\
\text { Calificación } 100 \%\end{array}$} & \multicolumn{2}{|c|}{ Calificación mediana } & \multirow{3}{*}{$\begin{array}{c}\mathbf{p}^{*} \\
<0.0001\end{array}$} \\
\hline & Antes & Después & \\
\hline Conocimiento general & $\begin{array}{c}44.5 \\
\text { RIC (37-48) }\end{array}$ & $\begin{array}{c}54 \\
\operatorname{RIC}(51-57)\end{array}$ & \\
\hline Procedimiento adecuado para la realización del método ITB & $\begin{array}{c}11 \\
\operatorname{RIC}(11-14)\end{array}$ & $\begin{array}{c}23 \\
\operatorname{RIC}(22-23)\end{array}$ & $<0.0001$ \\
\hline Evaluación e interpretación de cálculo del ITB & $\begin{array}{c}3 \\
\operatorname{RIC}(0-8)\end{array}$ & $\begin{array}{c}20 \\
\operatorname{RIC}(19-20)\end{array}$ & $<0.0001$ \\
\hline Evaluación total & $\begin{array}{c}60 \\
\operatorname{RIC}(51-68)\end{array}$ & $\begin{array}{c}97 \\
\operatorname{RIC}(94-100)\end{array}$ & $<0.0001$ \\
\hline
\end{tabular}

*Prueba de Wilcoxon.

EAP: enfermedad arterial periférica; ITB: índice tobillo-brazo; RIC: rango intercuartilar.

Resultados en las evaluaciones inicial y final

Para el total de participantes se encontró una diferencia altamente significativa $(\mathrm{p}<0.0001)$ entre la mediana del resultado global en la evaluación inicial (60; RIC: 51-68) y la evaluación final (97; RIC: 94-100). En la evaluación inicial aprobó (con más del $60 \%$ de respuestas correctas) el $60 \%$ ( $\mathrm{n}=32$ ), en comparación con el $100 \%$ $(n=54)$ en la evaluación final. En los resultados por área de conocimiento, también se encontró una diferencia significativa en las tres áreas ( $\mathrm{p}=0.0001)$, con una calificación $>90 \%$ en la evaluación final en el $97 \%$ $(\mathrm{n}=52)$ de los participantes (Tabla 2).

No se encontraron diferencias significativas por sexo en el porcentaje de participantes aprobados $(\mathrm{p}=0.338)$, pero en el grado de residencia médica sí hubo una diferencia significativa $(p=0.040)$ entre la mediana del resultado global en la evaluación, siendo el tercer grado el único que obtuvo una calificación mayor del 60\% en ambas evaluaciones en el 100\% $(n=14)$ de los residentes. En la evaluación final, todos los grados aprobaron la evaluación, con un porcentaje superior al 95\% (Fig. 1).

\section{DISCUSIÓN}

Puesto que los profesionales de la salud son los principales responsables del diagnóstico oportuno de la EAP, preocupa la deficiencia o ausencia en su diagnóstico, encontrándose deficiencias en el conocimiento tanto teórico como práctico, por lo que deben contar con capacitación adecuada e información actualizada para realizar una labor diagnóstica efectiva ${ }^{13}$.

En 2010, Wyatt, et al. ${ }^{9}$ realizaron en EE.UU. un estudio longitudinal no aleatorizado con 24 residentes de medicina interna para valorar los conocimientos de procedimiento para el ITB, por medio de un taller de entrenamiento con ayuda audiovisual. Al inicio del estudio el $4 \%$ de los participantes lo midió, el 10\% lo calculó y el $45 \%$ lo interpretó correctamente. Después del programa de capacitación se obtuvo un incremento en la adquisición de conocimientos, medición y cálculo, con un 50, 75 y $88 \%$, respectivamente. En nuestro estudio se encontraron también diferencias significativas posteriores al taller en las tres áreas que se evaluaron: conocimiento, procedimiento y evaluación e interpretación del cálculo, con una calificación superior al $94 \%$ en el $100 \%$ de los participantes.

En 2013, Georgakarakos, et al. ${ }^{10}$ realizaron un estudio en Grecia con cinco estudiantes de medicina, a los que les proporcionaron 26 h de capacitación en el método ITB (13 h de teoría y $13 \mathrm{~h}$ de práctica) con 18 pacientes con EAP de diferente gravedad, supervisados por cirujano vascular, y fueron comparados con un entrenador. Hubo respectivas evaluaciones del ITB de estudiantes frente a entrenador con diferencia en cifras de 0.32 frente a 0.23 


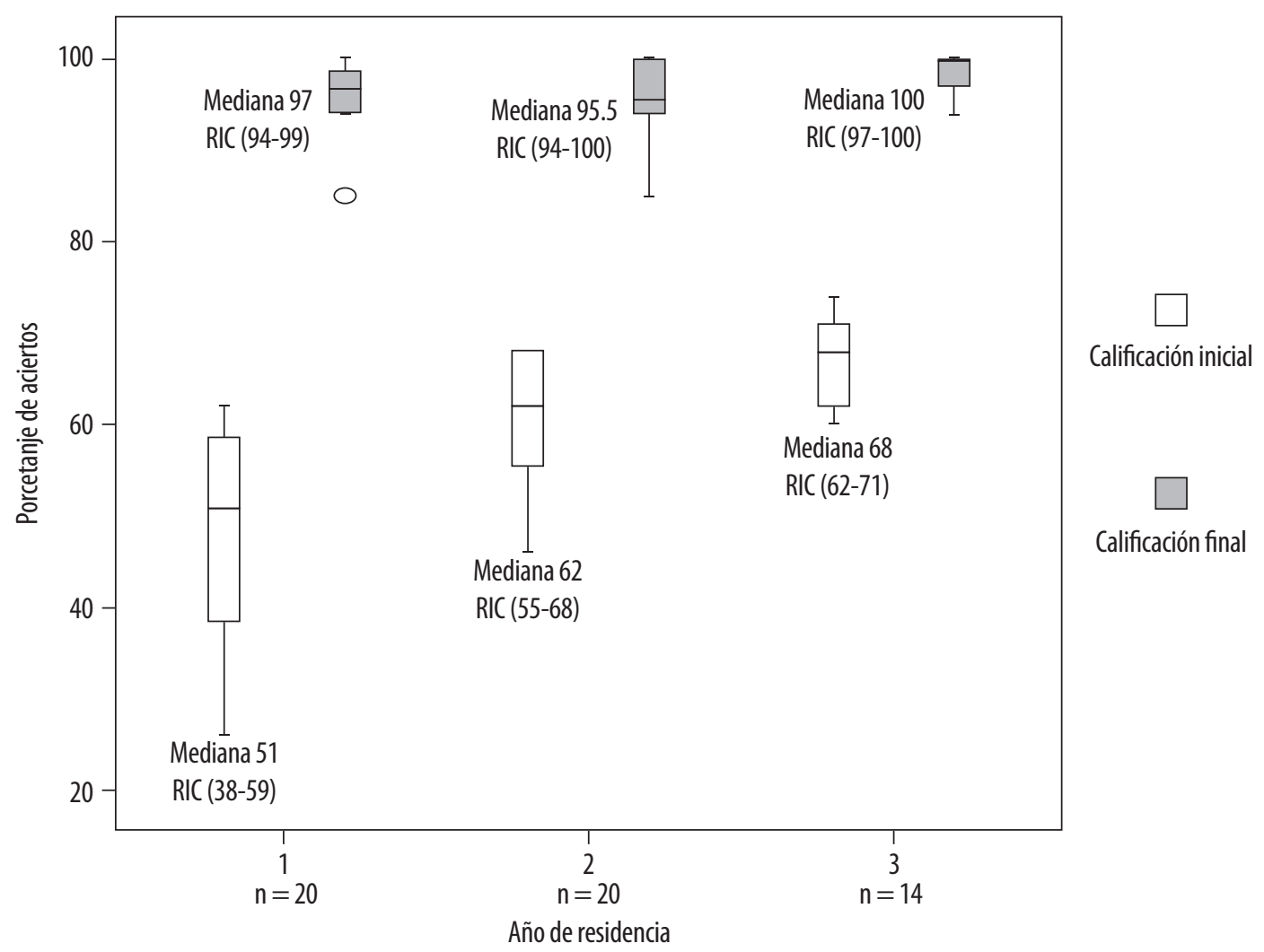

$p=0.040$ (prueba de Kruskal-Wallis).

Figura 1. Evaluación inicial y al final del taller de capacitación para el diagnóstico de la EAP por grado de residencia. EAP: enfermedad arterial periférica; RIC: rango intercuartilar.

$(\mathrm{p}=0.0002)$ para la EAP, logrando que al finalizar el taller no existieran diferencias entre alumnos y entrenador. En nuestro estudio la comparación realizada fue entre los grados de residencia de medicina familiar, y se encontró que los participantes del tercer año eran los que obtenían los porcentajes más altos de aprobación tanto en la evaluación inicial como en la final. Las diferencias por grado de residencia se explican por el conocimiento adquirido previamente, secundario a exposición en el Servicio de Angiología. El grado de residencia que presentó la mayor ganancia de conocimientos fue el de primer año, lo que concuerda con que, una vez acreditado el curso de capacitación, se incrementa e iguala el conocimiento con los otros grados de residencia.

En otro estudio realizado con 53 médicos generales en los Países Bajos en 2005,
Willigendael, et al. ${ }^{11}$, después de una capacitación de $4 \mathrm{~h}$ ( $2 \mathrm{~h}$ de teoría y $2 \mathrm{~h}$ de práctica) en un periodo de 6 meses, realizaron una comparación de estos médicos capacitados frente a un especialista vascular sin capacitación para el método ITB. Sus conclusiones fueron que aumentaron sus mediciones correctas del ITB de un $83 \%$ al final de la capacitación frente a un 35\% sin entrenamiento $(p=0.001)$.

En nuestro estudio no se realizó una comparación con un especialista vascular, pero se evaluó la realización del ITB en pacientes según una lista de cotejo avalada por la American College of Cardiology Foundation/American Heart Association. Tal evaluación se realizó después del taller de capacitación con supervisión del cirujano vascular, con una calificación $\geq 90$ en el $86 \%$ de los participantes, lo que demuestra 
que la mayoría realizó adecuadamente el método ITB en los pacientes.

Los resultados de la evaluación inicial en nuestro estudio ponen de manifiesto la falta de conocimiento de los participantes, que se puede explicar por la falta de lectura, exposición y rotación por el Servicio de Angiología, ya que el $40 \%$ obtuvo una calificación inferior al $60 \%$ en esta evaluación, la cual se incrementó después de la capacitación a más del $80 \%$ en la totalidad de los participantes. Además, aunque no fue un objetivo de nuestro estudio, se obtuvieron los siguientes resultados del ITB en los pacientes: $48 \%$ (26 pacientes) con ITB normal, $39 \%$ (21 pacientes) con EAP y $13 \%$ (7 pacientes) con calcificaciones de Mönckeberg. Para mejorar el resultado global se requiere que, en futuras capacitaciones, se refuercen los temas de conceptos generales sobre la EAP y el ITB, clasificación e interpretación de los resultados, y que los participantes realicen la lectura del material de ayuda antes del curso. Para garantizar la obtención de un aprendizaje significativo, traducido en una correcta evaluación del ITB en la atención diaria a los pacientes, también se requiere llevar a cabo la supervisión de la correcta aplicación del ITB por personal ya capacitado. Las limitaciones de nuestro estudio son no llevar un seguimiento de los pacientes ni evaluar los grados del ITB (leve, moderada y severa).

\section{CONCLUSIONES}

El taller tuvo un impacto positivo y significativo en la adquisición de conocimientos sobre el método ITB para el diagnóstico de la EAP en los residentes de medicina familiar que laboran en la atención primaria. La metodología descrita en esta capacitación permite incrementar el nivel de conocimientos adquiridos a través del curso, y generar recomendaciones y consejos para reforzar los conocimientos. El uso del ITB en este modelo de capacitación es adecuado y de fácil aplicación en las unidades de atención primaria para el diagnóstico oportuno de la EAP.

\section{BIBLIOGRAFÍA}

1. Fowkes FGR, Rudan D, Rudan I, Aboyans V, Denenberg JO, McDermott MM, et al. Comparison of global estimates of prevalence and risk factors for peripheral artery disease in 2000 and 2010: a systematic review and analysis. Lancet. 2013;382(9901):1329-40.

2. Tendera M, Aboyans V, Bartelink ML, Baumgartner I, Clément $D$, Collet JP, et al. Guía de práctica clínica de la ESC sobre diagnóstico y tratamiento de las enfermedades arteriales periféricas. Rev Esp Cardiol. 2012;65(2):1-57.

3. Lozano R, Gómez-Dantés H, Garrido-Latorre F, Jiménez-Corona A, Campuzano-Rincón JC, Franco-Marina F, et al. La carga de enfermedad, lesiones, factores de riesgo y desafíos para el sistema de salud en México. Salud Pública Mex. 2013;55:580-94.

4. Rooke TW, Hirsch AT, Misra S, Sidawy AN, Beckman JA, Findeiss $L$, et al. Management of patients with peripheral artery disease (compilation of 2005 and 2011 ACCF/AHA Guideline Recommendations): a report of the American College of Cardiology Foundation/American Heart Association Task Force on Practice Guidelines. J Am Coll Cardiol. 2013;61(14):1555-70.

5. Aboyans V, Criqui MH, Abraham P, Allison MA, Creager MA, Diehm $C$, et al. Measurement and interpretation of the ankle-brachial index: a scientific statement from the American Heart Association. Circulation. 2012;126(24):2890-909.

6. Gerhard-Herman MD, Gornik HL, Barrett C, Barshes NR, Corriere MA, Drachman DE, et al. 2016 AHA/ACC guideline on the management of patients with lower extremity peripheral artery disease: executive summary: a report of the American College of Cardiology/American Heart Association Task Force on Clinical Practice Guidelines. Circulation. 2017;135(12):e686-e725.

7. Mohler ER, Treat-Jacobson D, Reilly MP, Cunningham KE, Miani $\mathrm{M}$, Criqui $\mathrm{MH}$, et al. Utility and barriers to performance of the ankle-brachial index in primary care practice. Vasc Med. 2004;9:253-60.

8. Anderson JL, Halperin JL, Albert NM, Bozkurt B, Brindis RG, Curtis $\mathrm{LH}$, et al. Management of patients with peripheral artery disease (compilation of 2005 and 2011 ACCF/AHA guideline recommendations): a report of the American College of Cardiology Foundation/American Heart Association Task Force on Practice Guidelines. J Am Coll Cardiol. 2013;61:1555-70.

9. Wyatt MF, Stickrath C, Shah A, Smart A, Hunt J, Casserly P. Anklebrachial index performance among internal medicine residents. Vasc Med. 2010;15(2):99-105.

10. Georgakarakos E, Papadaki E, Vamvakerou V, Lytras D, Tsiokani A, Tsolakaki $O$, et al. Training to measure ankle-brachial index at the undergraduate level: can it be successful? Int J Low Extrem Wounds. 2013;12(2):167-71.

11. Willigendael EM, Teijink JA, Bartelink ML, Langenberg $M$, Welten RJ, Büller HR, et al. Combined training of GPs and practice-assistants on peripheral arterial disease: positive effects after six months. Fam Pract. 2005;22(5):520-2.

12. Hernández-Salazar VM, Mendoza-Rivera R. Enfermedad arterial periférica con y sin diabetes adscritos a la UMF 66. Trabajo de titulación para obtener el título de especialidad en Medicina Familiar. Universidad Veracruzana; 2014. Disponible en: https:// www.uv.mx/mf066/

13. Chaudru S, de Müllenheim PY, Le Faucher A, Kaladji ffA, Jaquinandi V, Mahé G. Training to perform ankle-brachial index: systematic review and perspectives to improve teaching and learning. Eur J Vasc Endovasc Surg. 2016;51(2):240-7. 